\title{
Adaptive Modulation for SFBC-OFDM Systems with Zero-Forcing Equalization
}

\author{
P.N. Kota \\ Research student \\ SPPU University \\ Pune, India
}

\author{
A.N. Gaikwad \\ ZEAL College Of Engg. \\ Nahre,Pune
}

\author{
Pranav M. Patil \\ E\&TC Department, \\ MES's College of Engineering \\ Pune, India
}

\begin{abstract}
The Space frequency block coded OFDM gives better performance in highly frequency selective and time varying channel than STBC OFDM. Hence this system can be mostly useful in vehicular communication where user is highly mobile. In this paper, An Adaptive modulation scheme for SFBC-OFDM system is proposed to give the reliable connectivity as well as efficient data rate to highly mobile user. Here, the bit rate is adapted according to the SNR value estimated from channel. For variation in bit rate the M-QAM modulations scheme is selected by comparing estimated SNR value and threshold value for switching. Also to reduce the effect channel which distorted the transmitted signal the Zero forcing (ZF) equalization is used. Finally performance of proposed system is analyzed with BER VS SNR plot.
\end{abstract}

\section{Keywords}

Orthogonal Frequency Division Multiplexing (OFDM), Space Frequency Block coding (SFBC), Space time block coding (STBC)

\section{INTRODUCTION}

Feature mobile communication must satisfies the growing demands of High data rate, reliable communication, low power consumption as well as spectral efficiency. In case of vehicular mobile communication, where the high mobility scenario occurs the wireless connection is not trusted or it fails to give expected data rates. In case of high mobility scenario, the received signal quality degrades to more extent due to the inter carrier interference (ICI) and inter symbol interference (ISI) problems. It happens because of time variation in channel due to mobile speed characterized by Doppler spread. Also antenna selection is the bigger problem in high speed vehicular communication. As the channel is frequency selective or fast fading the diversity is the best solution to the fast fading channels. Higher the diversity gain, lower the effect of ISI and ICI on the transmitted signal. Aloumoti STBC give better diversity gains[1]. Hence they play important role in today's wireless communication system along with OFDM . In STBC OFDM the replica of the signal is transmitted on the same subcarrier of two consecutive OFDM symbols. Hence, STBC gives better performance where the channel remains constant across two consecutive OFDM symbol duration [2]. Hence, it is useful in stationary scenario, where transmitter and receiver are stationary. But in vehicular communication due to high mobility the channel may not remains constant across two OFDM symbols duration. Hence STBC OFDM fails to give reliable connection. In this case SFBC OFDM gives better performance as in SFBC the replica of signal is transmitted on neighboring subcarrier of same OFDM symbol. It is assumed that channel remains constant across two consecutive subcarriers [3]. Along with the diversity there is need of good signal detector at the receiver. For receive diversity simple maximum-likelihood (SML) detector. However, this detector is optimum in quasi static channel only. Recently, Vielmonet al. recommended three novel detectors for time varying channel to the combat the effect of rapid channel variation. These detectors are zero forcing (ZF), joint maximum-likelihood (JML) and decisionfeed back (DF).Out of this the DF is used in this paper because of its low complexity [4].

A major drawback of the wireless vehicular communication system is deep fading due to high mobility of device. In SFBC OFDM the fixed modulation scheme is used which fails to mitigate the deep fading problem. These problems can be minimized by adapting the parameters like modulation scheme, power, and ranges of system parameters based on channel condition. In 1968 Hayes proposed power adaptive modulation scheme [5]. Adaptive scheme based on mode switching level is proposed by Hanzo et al [6-9] shows that it provides better spectral efficiency and BER performance than fixed modulation. For multicarrier system the adaptive modulation is performed by Kalet known as AOFDM [10]. Advantages of diversity technique along with AOFDM are investigated in [11].Hence, in this paper an adaptive modulation technique is proposed for SFBC OFDM system. In which the modulation schemes assigned to SFBC OFDM system are changed according to channel conditions. Channel condition is measured in terms of received SNR estimated from channel noise and AWGN noise. Also ZF detection technique is applied at receiver to retrieve the original signal. Finally the performance of the proposed adaptive modulation system is analyzed in terms of SNR VS BER plots. Also, performance is given for different detection schemes as mentioned above.

So this paper is arranged in following sections section 2 gives the Overview of SFBC OFDM SYTEM with simple SFBC encoding-decoding. In Section 3 review of signal equalization technique at receiver. Section 4 describes proposed adaptive modulation technique for SFBC-OFDM. In Section 5 the performance of SFBC OFDM system is analyzed with and without adaptive modulation Section 6 concludes this paper.

\section{SFBC-OFDM SYSTEM MODEL}

In Space time block coding, the symbols of an orthogonal design are transmitting on same subcarrier of subsequent OFDM symbol due to which performance of system degrades in fast channel variation in time. Hence, space frequency block coding is preferred to avoid problem of fast channel variation in time. In SFBC the symbols of 
orthogonal design transmitted on neighboring Subcarrier of same OFDM symbol. This is will illustrate in Figure1.

$$
\left[\begin{array}{l}
\mathrm{X}_{1} \\
\mathrm{X}_{2}
\end{array}\right] \rightarrow\left[\begin{array}{cc}
\mathrm{X}_{1} & \mathrm{X}_{2} \\
-\mathrm{X}_{2}^{*} & \mathrm{X}_{1}^{*}
\end{array}\right] \rightarrow \begin{gathered}
\text { space } \\
\text { subcarrier }
\end{gathered}
$$

Fig.1: Alamouti SFBC[2]

The example in Fig 2, show the basic idea behind the implementation of SFBC-OFDM system.

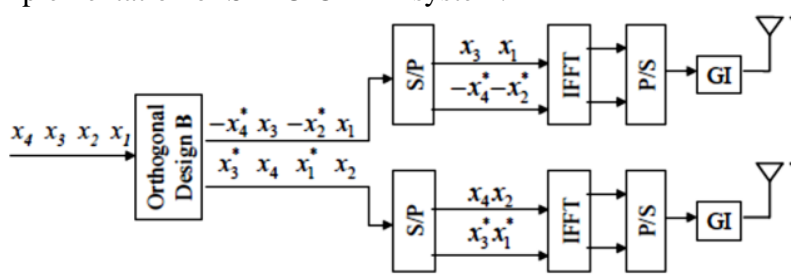

Fig.2: SFBC in OFDM With two Transmitters [2]

To examine SFBC-OFDM in more depth let us consider that $x_{i}(n)$ is $n^{\text {th }}$ signal transmitted from ith antenna then $x_{1}(n)$ is given as:

$$
\begin{aligned}
& \mathrm{x}_{1}(\mathrm{n})=\frac{1}{\sqrt{\mathrm{N}}} \sum_{\mathrm{k}=0}^{\mathrm{N}-1} \mathrm{x}_{1}(\mathrm{k}) \mathrm{W}_{\mathrm{N}}^{-\mathrm{nk}} \\
& n=0,1 . . N-1
\end{aligned}
$$

Where, $\mathrm{W}_{\mathrm{N}}^{-\mathrm{nk}}$ is twiddle factor, $x_{1}(n)$ is original QPSK signal to be transmitted Equation (1) can be written as

$$
\begin{array}{r}
\mathrm{x}_{1}(\mathrm{n})=\frac{1}{\sqrt{\mathrm{N}}} \sum_{\mathrm{m}=0}^{\left[\frac{\mathrm{N}}{2}-1\right]}\left[\mathrm{x}_{1}(2 \mathrm{~m})+\mathrm{W}_{\mathrm{N}}^{-\mathrm{n}} \mathrm{x}_{1}(2 \mathrm{~m}+1)\right] \mathrm{W}_{\left(\frac{\mathrm{N}}{2}\right)}^{-\mathrm{nm}} \\
=\frac{1}{\sqrt{2}}\left[x^{e}(n)+\mathrm{W}_{\mathrm{N}}^{-\mathrm{n}} x^{o}(n)\right](2)
\end{array}
$$

Where, $x^{e}(n) \frac{\mathrm{N}}{2}$ points IFFT of even samples of $\mathrm{x}_{1}(\mathrm{n})$ and $x^{o}(n)$ corresponds to odd samples of $\mathrm{x}_{1}(\mathrm{n})$.

Similarly for second antenna

$$
\begin{gathered}
x_{2}(n)=\frac{1}{\sqrt{N}} \sum_{k=0}^{N-1} x_{2}(k) W_{N}^{-n k} \\
\mathrm{x}_{2}(\mathrm{n})=\frac{1}{\sqrt{\mathrm{N}}} \sum_{\mathrm{m}=0}^{\left[\frac{\mathrm{N}}{2}-1\right]}\left[\mathrm{x}_{2}(2 \mathrm{~m})+\mathrm{W}_{\mathrm{N}}^{-\mathrm{n}} \mathrm{x}_{2}(2 \mathrm{~m}+1)\right] \mathrm{W}_{\left(\frac{\mathrm{N}}{2}\right)}^{-\mathrm{nm}}
\end{gathered}
$$

Now applying Alamouti scheme to adjacent carriers we have

$$
\begin{aligned}
\mathrm{X}_{1}(\mathrm{n}) & =\left[\mathrm{X}_{1}(0), \mathrm{X}_{1}(1), \mathrm{X}_{1}(2) \ldots \mathrm{X}_{1}(\mathrm{~N}-1)\right] \\
\mathrm{X}_{2}(\mathrm{n}) & =\left[-\mathrm{X}_{1}^{*}(1), \mathrm{X}_{1}^{*}(0),-\mathrm{X}_{1}^{*}(3)-\mathrm{X}_{1}^{*}(\mathrm{~N}-1)\right] \\
\mathrm{X}_{2}(\mathrm{n}) & =\frac{1}{\sqrt{\mathrm{N}}} \sum_{\mathrm{m}=0}^{\left[\frac{\mathrm{N}}{2}-1\right]}\left[-\mathrm{X}_{1}^{*}(2 \mathrm{~m}+1)+\mathrm{W}_{\mathrm{N}}^{-\mathrm{n}} \mathrm{X}_{1}^{*}(2 \mathrm{~m})\right] \mathrm{W}_{\left(\frac{\mathrm{N}}{2}\right)}^{-\mathrm{nm}} \\
& =\frac{1}{\sqrt{2}}\left[x^{e}(n)+\mathrm{W}_{\mathrm{N}}^{-\mathrm{n}} x^{o}(n)\right]
\end{aligned}
$$

$\mathrm{CP}$ is appended at the head of each symbol to eliminate ISI making all channel matrices circulant $\left(H_{i}\right)$. Received symbol after removal of $\mathrm{CP}$ is given by

$$
\mathrm{y}=\frac{1}{\sqrt{2}}\left[\mathrm{H}_{1} \mathrm{x}_{1}+\mathrm{H}_{2} \mathrm{x}_{2}+\mathrm{n}\right]
$$

Similarly, if two transmitter and receivers are used theneq(3) becomes

$$
\left[\begin{array}{l}
\mathrm{y}_{1} \\
y_{2}^{*}
\end{array}\right]=\frac{1}{\sqrt{2}}\left[\begin{array}{cc}
h_{11} & -h_{12} \\
h_{22}^{*} & h_{21}^{*}
\end{array}\right]\left[\begin{array}{l}
x_{1} \\
x_{2}^{*}
\end{array}\right]+\left[\begin{array}{l}
n_{1} \\
n_{2}^{*}
\end{array}\right]
$$

In equation (2) $h_{i j}, i, j \in\{1,2\}$ denotes the complex channel response at OFDM subcarrier $i$ from transmit antenna j. $n_{1}$ and $n_{2}$ are independent identically distributed (i.i.d) zero-mean complex Gaussian Circularly symmetric with variance $\sigma_{n}^{2}$ that represents the additive noise term. Each subcarrier assumed to experiences the frequency flat Rayleigh fading.

Now estimated channel response can be modeled as

$$
\widehat{H}=H+\left[\begin{array}{cc}
e_{11} & -e_{12} \\
e_{22}^{*} & e_{21}^{*}
\end{array}\right](5)
$$

Where, $\mathrm{E}$ is random matrix where elements $e_{i j}$ are i.i.d zeromean complex Gaussian circularly symmetric with variance $\sigma_{e}^{2}$.

At, receiver side the linear decoding of SFBC signal is done as [12]

$$
\begin{aligned}
& \mathrm{z}=\frac{1}{\sqrt{2}} \widehat{\mathrm{H}}^{\mathrm{H}} \mathrm{y} \\
& =\frac{1}{\sqrt{2}}\left(H^{H}+E^{H}\right)\left(\frac{1}{\sqrt{2}} H x+n\right) \\
& =\frac{1}{2}\left[\begin{array}{cc}
\left|h_{11}\right|^{2}+\left|h_{22}\right|^{2} & h_{21}^{*} h_{22}^{*}-h_{11}^{*} h_{12}^{*} \\
h_{21}^{*} h_{22}^{*}-h_{11}^{*} h_{12}^{*} & \left|h_{21}\right|^{2}+\left|h_{12}\right|^{2}
\end{array}\right] x \\
& +\frac{1}{2} \mathrm{E}^{\mathrm{H}} \mathrm{Hx}+\frac{1}{\sqrt{2}}\left(H^{H}+E^{H}\right) \mathrm{n} \\
& =\frac{1}{2}\left[\begin{array}{l}
\left(\left|\mathrm{h}_{11}\right|^{2}+\left|\mathrm{h}_{22}\right|^{2}\right) \mathrm{x}_{1} \\
\left(\left|\mathrm{~h}_{21}\right|^{2}+\left|\mathrm{h}_{12}\right|^{2}\right) \mathrm{x}_{2}^{*}
\end{array}\right] \\
& +\left[\begin{array}{l}
\left(\mathrm{h}_{21}^{*} \mathrm{~h}_{22}^{*}-\mathrm{h}_{11}^{*} \mathrm{~h}_{12}^{*}\right) \mathrm{x}_{2}^{*} \\
\left(\mathrm{~h}_{21}^{*} \mathrm{~h}_{22}^{*}-\mathrm{h}_{11}^{*} \mathrm{~h}_{12}^{*}\right) \mathrm{x}_{1}
\end{array}\right]+\frac{1}{2} \mathrm{E}^{\mathrm{H}} \mathrm{Hx} \\
& +\frac{1}{\sqrt{2}}\left(\mathrm{H}^{\mathrm{H}}+\mathrm{E}^{\mathrm{H}}\right) \mathrm{n}(5 \mathrm{c})
\end{aligned}
$$

Where, $\mathrm{A}^{\mathrm{H}}$ is hermitian transpose of A. The term in (5a) is desired component, $(5 \mathrm{~b})$ leads to the self interference terms and $(5 \mathrm{c})$ denotes the additive noise terms.

material on each page should fit within a rectangle of $18 \mathrm{x}$ $23.5 \mathrm{~cm}$ (7" x 9.25"), centered on the page, beginning 2.54 $\mathrm{cm}$ (1") from the top of the page and ending with $2.54 \mathrm{~cm}$ (1") from the bottom. The right and left margins should be $1.9 \mathrm{~cm}\left(.75^{\prime \prime}\right)$. The text should be in two $8.45 \mathrm{~cm}(3.33 ")$ columns with a $.83 \mathrm{~cm}(.33 ")$ gutter.

\section{EQUALIZATION TECHNIQUE}

In MIMO the ISI occurs due to the multipath propagation. This distorted the transmitted signal which in turn causes bit error rate at receiver. Hence, equalizer is used to minimize the error between actual output and desired output by continuous updating its filter coefficient. Equalization can be used in both frequency and time domain. In this paper Zero forcing equalization method is implemented in frequency domain. 


\subsection{Zero forcing equalization}

In Zero-forcing equalization the coefficient are chosen to force the samples of the combined channel and equalizer impulse response to zero. [13] The combined impulse response of the channel is given by

$$
H_{C H}(f) H_{e q}(f)=1
$$

Where, $H_{C H}(f)$ is folded frequency response of channel and $H_{e q}(f)$ is frequency response of equalizer. Zero forcing equalizer can be realized by multiplying equation (5b) by vector $1 / H(K)$ where $\mathrm{H}(\mathrm{K})$ is normalized MIMO-channel vector which can be formed as:

$$
\mathrm{H}=\mathrm{h}_{21}^{*} \mathrm{~h}_{22}^{*}-\mathrm{h}_{11}^{*} \mathrm{~h}_{12}^{*}
$$

In this case the equalization filter compensates for the channel induced ICI as well as ISI brought about by the transmitter and receiver filters. Zero forcing equalizer designed above does not eliminate all ISI because the filter is of finite length.

\section{PROPOSED SCHEME ADAPTIVE MODULATION FOR SFBC-OFDM}

The allocation of bits to the subcarriers SFBC-OFDM system can be uniform or non uniform. In frequency selective fading channel some subcarriers undergo deep fade while some undergo less fading. The occurrence of bit errors is due to the deep fading of sub channels. To minimize the occurrence of bit errors at deeply faded subcarriers, one can change the bits allocated to those subcarriers i.e allocate less bits than pervious. This can be done by changing the modulation scheme used for transmission [14]. In this system 8QAM, 16QAM, 32QAM and 64QAM modulation schemes are used. The change in modulation scheme depends on estimated value of SNR from channel at receiver and threshold value for switching. The SNR estimation at receiver is given as

$$
\bar{\gamma}=\gamma_{0} \sum_{l=1}^{L} E\left\{\|H(l)\|_{F}^{2}\right\}
$$

Where, $\|H(l)\|_{F}^{2}=\operatorname{Tr}\left(H H^{*}\right)=\operatorname{Tr}\left(H_{w}^{*} \wedge_{R} H_{w} \wedge_{T}\right)$ is the frobenius norm, $\mathrm{L}$ is the number of path.

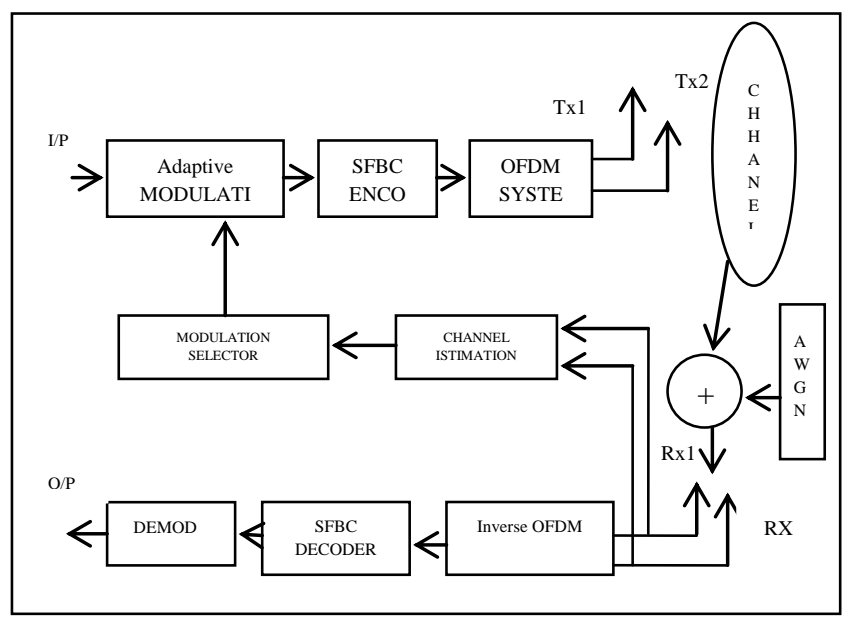

Fig 3.Praposed adaptive modulation Scheme

By using total transmitted power $\left(P_{T}\right)$ and utilized bandwidth (B), [15] average SNR $\gamma_{0}$ per receive antenna under unity channel gain is given as

$$
\gamma_{o}=\frac{P_{T}}{B \sigma_{n}^{2}}
$$

Where, $\sigma_{n}^{2}=N_{0}$ under the assumption of circular symmetric complex Gaussian random variables and $N_{0} / 2$ is the power spectral density of real/imaginary component of noise. Fig 3 show the block schematic for proposed SFBC-OFDM system with adaptive modulation.

One can also detect the SNR value by computing the power of wanted signal divided by the power of noise. But from this SNR before noise reduction is computed.. To get the SNR after noise reduction, Residual noise power needs to be computed and it is found as follows.

SNR before noise reduction $=\sum \frac{\text { power of wanted signal }}{\text { power of noise }}(10)$

Residual noise $=$ Actual signal - noise reduced signal SNR after noise reduction $=\sum \frac{\text { power of wanted signl }}{\text { power of residual noise }}$

(11)

The estimated value of SNR in (8) or (11) at receiver is used for selection of modulation scheme at transmitter. This estimated SNR at receiver is feed back to transmitter. At transmitter the estimated SNR and threshold are compared and based on this comparison the modulation scheme is selected. Table 1 shows the SNR value range and respective thresholds for modulation scheme selection

The capacity of the channel is estimated using the below equation

$\mathrm{C}=\mathrm{Blog}_{2}(1+\mathrm{SNR})$

Where B is the bandwidth of transmitted signal and SNR is the estimated SNR of received signal [16].

Table 1: Modulation schemes and SNR thresholds

\begin{tabular}{|c|c|}
\hline MODULATION & THRESHOLD \\
\hline 4QAM & $0 \leq \mathrm{SNR}<6$ \\
\hline 8QAM & $6 \leq \mathrm{SNR}<10.5$ \\
\hline 16QAM & $10.5 \leq \mathrm{SNR}<13.5$ \\
\hline 32QAM & $13.5 \leq \mathrm{SNR}<16.6$ \\
\hline 64QAM & $16.6 \leq \mathrm{SNR}$ \\
\hline
\end{tabular}

\section{SIMULATION ANDDISCUSSION}

At transmitter initially input signal is 32QAM modulated and passed through SFBC-OFDM system. Then output of SFBCOFDM system transmitted on frequency-selective Rayleigh fading channel. At the receiver, the strength of received signal is calculated and channel estimation is done. Zero forcing Channel equalization method is used to remove the effect of ISI. The received SNR is calculated using the signal

Strength and channel estimation. The estimate selects the appropriate constellation size of M-QAM signaling that suits the channel condition. The data rate is adapted by the transmitter by changing modulation level based on the received SNR. The flow chart is for the simulation is shown in fig (5). 


\subsection{SIMULATION PARAMETERS}

Table (2) gives the simulation parameters which are taken from slandered wireless data. The simulation is done as per the flow chart shown in fig (3).At first the bit stream for

\begin{tabular}{|l|l|}
\hline \multicolumn{1}{|c|}{ Parameters } & \multicolumn{2}{c|}{ Type } \\
\hline Modulation & M-QAM (M=4,8,16,32,64) \\
\hline $\begin{array}{l}\text { MIMO antenna } \\
\text { configuration }\end{array}$ & $2 \times 2$ \\
\hline Signal to noise ration & $0: 5: 40 \mathrm{~dB}$ \\
\hline Average transmit power & $1 \mathrm{db}$ \\
\hline Fading & $\begin{array}{l}\text { Frequency selective Rayleigh } \\
\text { fading }\end{array}$ \\
\hline Carrier frequency & $900 \mathrm{MHz}$ \\
\hline Bandwidth of signal(Bs) & $200 \mathrm{MHz}$ \\
\hline Symbol period & $1 / \mathrm{Bs}$ \\
\hline NO of paths & 12 \\
\hline
\end{tabular}

transmission is generated. Then this stream is modulated with M-QAM modulation scheme and symbols are formed. These symbols are encoded using SFBC encoder. Then OFDM modulation is performed on each SFBC encoded stream. Finally generated OFDM symbols are transmitted via frequency selective Rayleigh fading channel. At receiver the SFBC encoded signal is detected using the Zero forcing detector. Then it is demodulated and decoded to get original signal back.BER and SNR are calculated.

\section{Table 2: Simulation parameters and Constraints}

The calculated SNR is feed back to the transmitter to select proper modulation Scheme.

\subsection{RESULTS AND DISCUSSION}

In analysis SFBC-OFDM system with adaptive modulation. The results are derived for Channel capacity vs SNR and BER vs SNR. Fig 4 gives the capacity of channels for 8 QAM,16 QAM,32 QAM and 64 QAM modulations under frequency selective Rayleigh fading channel. Fig 6 gives the capacity of channel for the adaptive modulation technique.

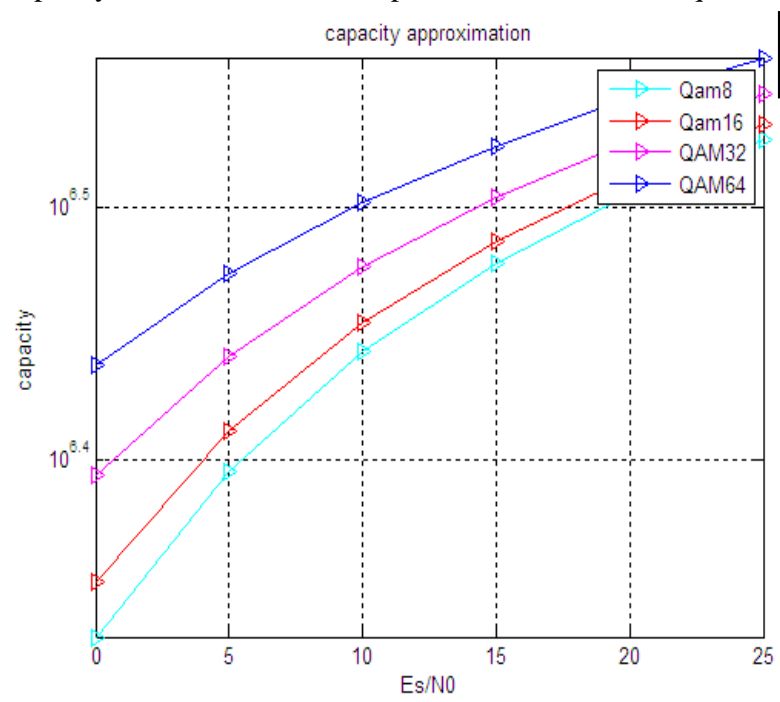

Use next SNR

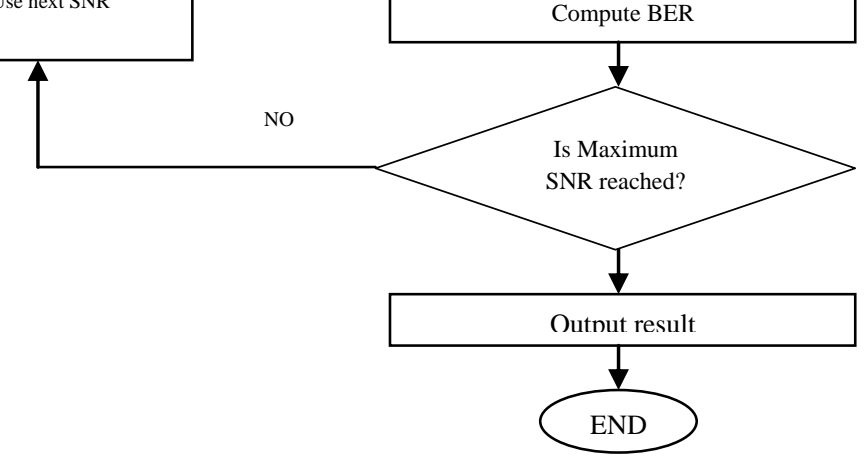

Fig 5: Flow chart for simulation of adaptive SFBCOFDM system

Fig 4: Capacity variation with SNR for different modulation scheme 


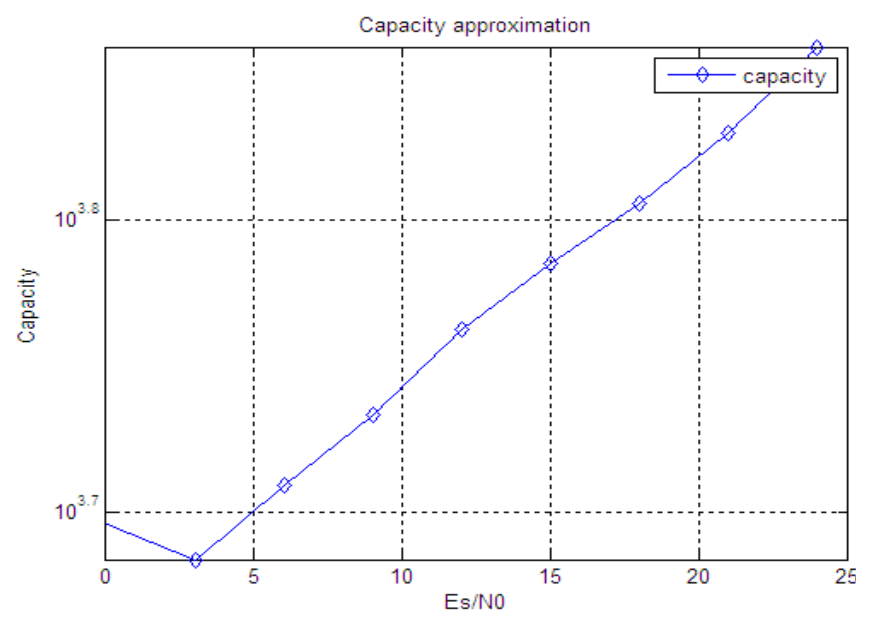

Fig 6 :BER performance of different modulation schemes

In Fig 7 the BER VS SNR plot for SFBC OFDM system with 8QAM, 16 QAM, 32 QAM and 64 QAM are obtained.from fig it is seen that as the modulation order increases the BER also increases.But as the SNR increases then BER decreases for every modulation order. Fig 8 gives the BER performance of SFBC-OFDM system with Adaptive modulation.Here the plotted curve seems like avarage of all curves for different modulation order in fig 7 .

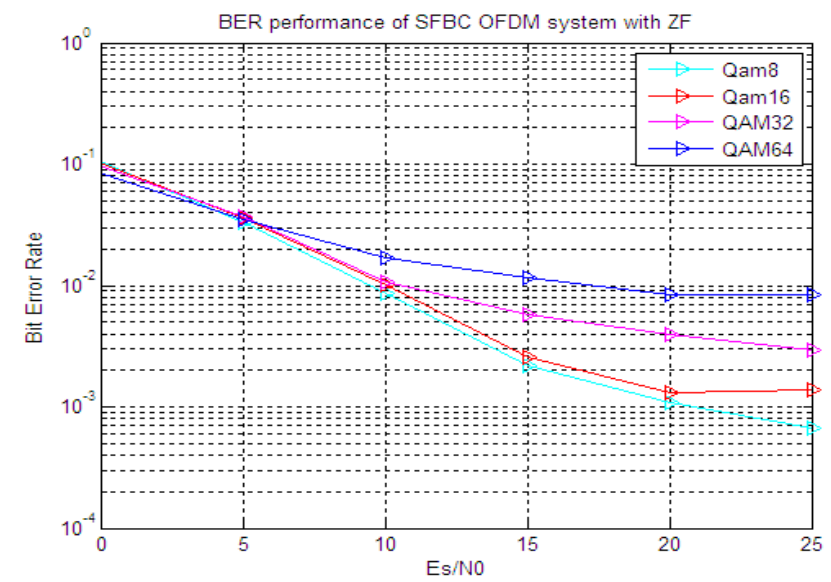

Fig 7 : Performance of SFBC-OFDM system with different modulation scheme

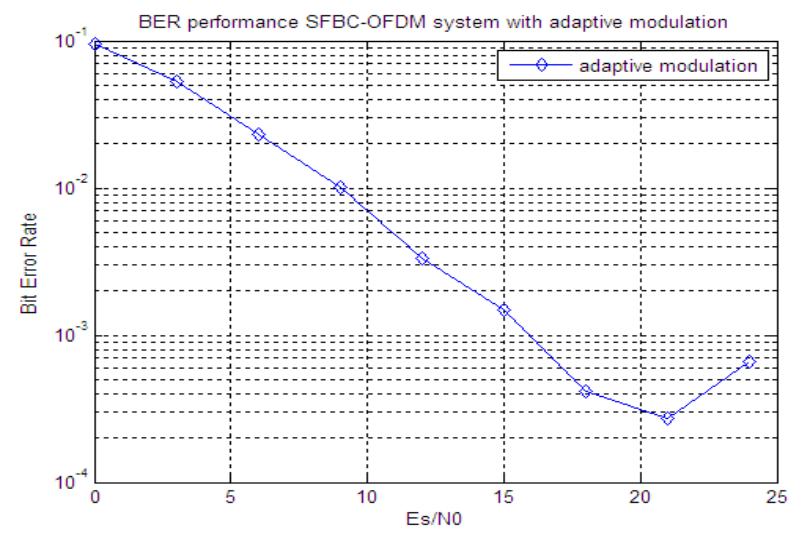

Fig 8: Performance of SFBC-OFDM system with adaptive modulation

\section{CONCLUSION}

In this paper the capacity vs SNR and BER vs SNR performance of proposed adaptive modulation scheme for SFBC-OFDM scheme is analyzed. For lower SNR values the capacity of channel is very low. Hence lower order modulation scheme like 8 QAM gives less BER for Low SNR values. Hence it helps in maintaining the connectivity in very bad channel conditions. But when channel conditions are good then received SNR values are high. At this time the capacity of channel is also high. Here 8QAM again gives very low BER but data rate is not achieved as channel allows more data rate.. So for achieving data rate in good channel conditions the higher modulation order are used.

Adaptive modulation gives the average performance which helps in achieving data rate as well as reliable connectivity.

Please use a 9-point Times Roman font, or other Roman font with serifs, as close as possible in appearance to Times Roman in which these guidelines have been set. The goal is to have a 9-point text, as you see here. Please use sans-serif or non-proportional fonts only for special purposes, such as distinguishing source code text. If Times Roman is not available, try the font named Computer Modern Roman. On a Macintosh, use the font named Times. Right margins should be justified, not ragged.

\section{REFERENCES}

[1] S.M. Alamouti, "A simple transmit diversity technique for wireless communications," IEEE J. Sel. Areas Common., vol.16, no.8, pp.1451-1458, 1998.

[2] G. Bauch, "Space-time block codes versus spacefrequency block codes," in Proc. IEEE Vehicular Technology Conference, pp. 567- 571, May 2003

[3] Lee, K.F. Williams, D.B. "A space-frequency transmitter diversity technique for OFDM systems" Global Telecommunications Conference, 2000. GLOBECOM '00. IEEE
10.1109 /GLOCOM.2000.891885

[4] Mohammad AftabAlam Khan, MehwashFarooqui "Performance Analysis of SFBC-OFDM System with Frequency Domain Equalization" International Journal of Scientific \& Engineering Research, Volume 3, Issue 11 , November-2012

[5] Hayes JF. Adaptive feedback communications. IEEE Trans CommunTechnol 1968;16:29-34.

[6] Torrance J, Hanzo L. Optimisation of switching levels for adaptive modulation in slow rayleigh fading channel. Electronic Letters 1996;32:1167-9

[7] Choi BJ, Hanzo L. Optimum mode-switching assisted adaptive modulation. In: Proceedings of IEEE global telecommunication Conference (GLOBECOM'01), vol. 6,2001, p. 3316-20.

[8] Hanzo L, Web W, Keller T. Single and multicarrier quadrature amplitude modulation. New York, USA: Wiley; 2000.

[9] Keller T, Hanzo L. Adaptive muticarriermodulation:a convenient frame work for time-frequency processing wireless communications. Proc IEEE 2000;88:611-40.

[10] Kalet I. The multitone channel. IEEE Trans Commun 1989;37:119-24 
[11] Liew TH, Hanzo L. Space-time block coded adaptivemodulation aided OFDM. In: Proceedings of IEEE globaltelecommunication conference (GLOBECOM'01) vol. 1,2001. p. 136-40.

[12] Liang Heng, Member, Louay M.A. Jalloul“Performance of the 3GPP LTE Space-FrequencyBlock Codes in Frequency-Selective Channels withImperfect Channel Estimation"DOI 10.1109/TVT.2014.2341612, IEEE Transactions on Vehicular Technology.

[13] Mohammad AftabAlam

Khan, MehwashFarooqui"Performance Analysis of SFBCOFDM System with Frequency Domain Equalization"International Journal of Scientific \& Engineering Research, Volume 3, Issue 11, November2012

[14] R.O. Abolade,Z. K. Adeyemo"Adaptive Modulation Performance In MimoOSTBC Over Rayleigh Fading
Channel"International Journal of Engineering \& Computer Science IJECS -IJENS Vol:12 No:06

[15] Jinliang Huang Svante Signell“On Performance of Adaptive Modulation in MIMOSystems Using Orthogonal Space-Time Block Codes"IEEE TRANSACTIONS ON VEHICULAR TECHNOLOGY, VOL. 58, NO. 8, OCTOBER 2009

[16] VarshaBirla,Rupesh Dubey "Capacity Enhancement of WiMAX System Using Adaptive Modulation and Code Rate" International Journal of Emerging Technology and Advanced Engineering Website: www.ijetae.com (ISSN 2250-2459, Volume 2, Issue 8, August 2012)

[17] Mohammad Torabi“'Adaptive modulation for spacefrequency block coded OFDM systems"Int. J. Electron. $\begin{array}{llll}\text { Commun. (AEÜ) } 62 \quad \text { (2008) } & 521\end{array}$ 533www.elsevier.de/aeue 